\title{
The Rahman-Nat diffraction on a thin laser-induced grating of the refractive index in semiconductors
}

\author{
O.Yu. Semchuk, O.I. Gichan, L.G. Grechko \\ A. Chuiko Institute of Surface Chemistry, NAS of Ukraine, \\ 17, General Naumov str., 03164 Kyiv, Ukraine \\ Fax: (380-44)424-3567; e-mail: semchuk_oleksandr@isc.gov.ua
}

\begin{abstract}
Studied in this work is the Rahman-Nat diffraction on a thin grating of refractive index in semiconductor, which was created by an interference pattern of two coherent laser beams. Numeral calculations showed that the maximal diffraction efficiency for the Rahman-Nat diffraction in the first order was $28.2 \%$ for a purely phase grating at its thickness $z \sim 200$, and $4.8 \%$ for a purely amplitude grating. It was also ascertained, that waves belonging to the nearby diffraction orders differ from each other by $\pi / 2$ in their phase.
\end{abstract}

Keywords: Rahman-Nat diffraction, refractive index, coherent laser beams, semiconductor, laser-induced grating, free carrier, phase grating, amplitude grating.

Manuscript received 14.04.09; accepted for publication 10.09.09; published online 30.10.09.

\section{Introduction}

Different types of surface structures created by laser radiation are of great interest in science and technique. For the first time, these structures were observed 30 years ago at the surface of semiconductors $\mathrm{Ge}$ and $\mathrm{Si}$ under the ruby laser pulse radiation [1]. Then similar structures were observed not only in semiconductors but also in metals $(\mathrm{Ni}, \mathrm{Cu}, \mathrm{Pb}, \mathrm{Al}$, steel, brass) and dielectrics ( $\mathrm{NaCl}$, fused and crystalline quartz). The special interest is caused by the laser-induced spatially periodic surface structures. As a rule, they are created at interference of a few laser beams on solid surface [2-10]. This technology has already found practical application, in particular, during melting metals [6], and making printing plates [11].

Spatially periodic structure created on a condensed matter surface can substantially change its optical properties. In particular, an interference pattern on semiconductor surface produces the laser-induced grating of the refraction index [12]. The optical parameters of the condensed matter on the surface of which an interference pattern is formed change in accordance with the division of interference pattern intensity $J$. Thus, a medium in which spatially periodic distribution of refractive index $N$ (or absorption coefficient $\alpha$ ) is created can come forward as a special diffraction grating.

In this work, the Raman-Nat diffraction on the thin grating of refraction index in semiconductor, which created by interference pattern of two coherent laser beams was studied. Numeral calculations of the diffraction efficiency inherent to this grating were carried out. In particular, shown is that the maximal diffraction efficiency for the Rahman-Nat diffraction of the first order is equal to $28.2 \%$ for a purely phase grating with the thickness $z \sim 200$, and $4.8 \%$ for a purely amplitude grating. It is also worth mentioning that waves belonging to the nearby orders of diffraction differ from each other by $\pi / 2$ phase in their phase.

\section{The laser-induced surface structures}

Up o recent years, to form laser-induced surface structures in solids, interference of two or more laser beams has been used [4-10]. In practice, a system of the interference beams can be formed by both one source with a light divider and a proper system of mirrors or slits and by a bunch (a grating) of the identical lasers [13]. We consider the first case, when an interference pattern is created by one source. A point source of light is assumed to be so far from an observer that its wave front can be considered as a flat propagating electromagnetic wave

$$
\vec{E}(\vec{r}, t)=\vec{A} \sin \left(\omega t-\vec{k} \vec{r}+\varphi_{0}\right),
$$

where $\omega$ - wave frequency, $\vec{k}$ - wave vector, $\varphi_{0}-$ initial phase. A source of light is regarded also as monochromatic, i.e. it radiates light of only one frequency $\omega$. Dividing this light beam by two ones (for 
example, using two closely located slits or a light divider with semitransparent mirrors in its way) and orienting them at the angle of $2 \theta_{0}$ to each other result in a spatially periodic modulated pattern of the intensity. In the places where the phase difference of these two beams is multiple of $2 \pi$, the amplitudes of the electric field intensity of both beams will be summed, i.e. the oscillations will strengthen each other, and we will see light stripes bright enough. In those places, where the phase difference is multiple an odd number of $\pi$, we will see dark stripes (Fig. 1).

Now a short mathematical description of the interference phenomenon will be given. Let us consider that each of these interfering waves is described by the expression (1), for the total field which is created as their sum, the following expression will be valid:

$$
\begin{aligned}
& \vec{E}(\vec{r}, t)=\vec{A}_{1} \sin \left(\omega t-\vec{k}_{1} \vec{r}+\varphi_{1}^{0}\right)+ \\
& +\vec{A}_{2} \sin \left(\omega t-\vec{k}_{2} \vec{r}+\varphi_{2}^{0}\right)+\ldots= \\
& =\sum_{i} \vec{A}_{i} \sin \left(\omega t-\vec{k}_{i} \vec{r}+\varphi_{i}^{0}\right) .
\end{aligned}
$$

When $i=2$, from the expression (2) it follows that

$$
\begin{aligned}
& |A|=\sqrt{A_{1}^{2}+A_{2}^{2}+2 A_{1} A_{2} \cos \left(\varphi_{1}-\varphi_{2}\right)}, \\
& \varphi=\operatorname{arctg} \frac{A_{1} \sin \varphi_{1}+A_{2} \sin \varphi_{2}}{A_{1} \cos \varphi_{1}+A_{2} \cos \varphi_{2}} .
\end{aligned}
$$

Using (2), the total light intensity of these two interfering beams can be written as

$$
\begin{aligned}
& J=\frac{c N}{4 \pi}\left\langle E^{2}\right\rangle= \\
& =J_{0}\left\{1+\frac{J_{\text {max }}-J_{\text {min }}}{J_{\text {max }}+J_{\text {min }}} \cos \left[\left(\vec{k}_{1}-\vec{k}_{2}\right) \vec{r}+\varphi_{2}^{0}+\varphi_{1}^{0}\right]\right\},
\end{aligned}
$$

where $\quad J_{0}=\frac{c N}{8 \pi}\left(A_{1}^{2}+A_{2}^{2}\right), \quad J_{\max }=\frac{c N}{8 \pi}\left(A_{1}+A_{2}\right)^{2}$, $J_{\min }=\frac{c N}{8 \pi}\left(A_{1}-A_{2}\right)^{2}$, and a bracket \langle\rangle means averaging over the time much greater than $\omega^{-1}$.

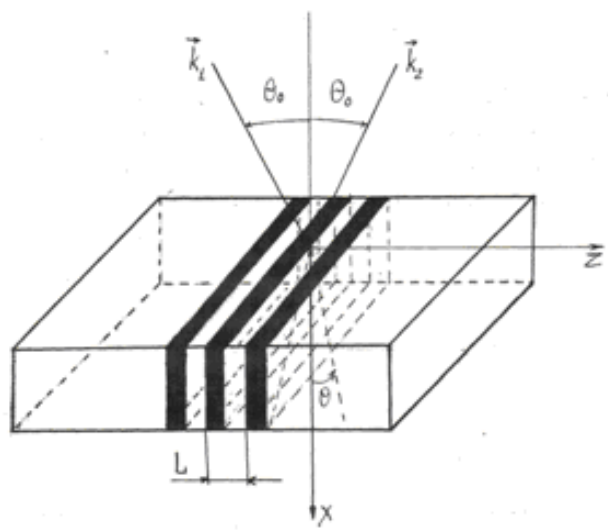

Fig. 1. Schematic presentation of the interference picture from two symmetrically-oriented beams $\theta_{0}$ and $\theta$ are the angles of the beam convergence in the air and in the semiconductor material).
The distance $L$ between neighbor stripes with the maximal intensity (the period of the interference pattern) for these two beams that converge at the angle of $2 \theta_{0}$ (Fig. 1) is given as

$$
L=\frac{\lambda}{2 \sin \theta} \text {. }
$$

Let us carry out the calculation of the interference pattern on the $x, y$ plane. Instead of the formula (2), the similar expression for the total electric field intensity forming on the $x, y$ plane as a result of the flat wave summing can be written as

$$
\begin{aligned}
& E(x, y)=\sum_{i=1}^{n} E_{i}+ \\
& +\sum_{i=1}^{n} E_{i}^{0} \exp \left\{-i k \sin \alpha_{i}\left(x \cos \beta_{i}-y \sin \beta_{i}\right)\right\},
\end{aligned}
$$

where $\alpha_{i}$ is the angle between the $i$-beam wave vector and the normal to the plane, $\beta_{i}$ - angle between the $i$ beam wave vector projection on the plane $(x, y)$ and the $(i+1)$-beam projection on this plane, $\lambda$ - laser radiation wavelength, $k=2 \pi / \lambda$ - wave vector.

For the interference of two beams $(i=2)$, we have one dimensional linear interference pattern. Indeed, assuming that

$E_{01}=E_{02}=E_{0}, \alpha_{1}=\alpha_{2}=\alpha, \beta_{1}=0, \beta_{2}=\pi$,

the intensity of this interference pattern can be written as follows

$$
J=\left|E_{0}\right|^{2}=4 E_{0}^{2} \cos (k x \sin \alpha) \text {. }
$$

For the interference of three beams $(i=3)$ from the equation (6), with the assumption that $E_{01}=E_{02}=E_{0}, \alpha_{1}=\alpha_{2}=\alpha_{3}=\alpha, \beta_{1}=0, \beta_{2}=\frac{2 \pi}{3}$,

$\beta_{3}=-\frac{2 \pi}{3}$

we have the following expression for the interference pattern intensity

$$
I=E_{0}^{2}\left[\begin{array}{c}
\left(-\cos (k x \sin \alpha)-\cos \left(k \sin \alpha\left(-\frac{x}{2}-y \frac{\sqrt{3}}{2}\right)\right)-\right)^{2}+ \\
-\cos \left(k \sin \alpha\left(-\frac{x}{2}+y \frac{\sqrt{3}}{2}\right)\right) \\
+\left(\begin{array}{c}
-\sin (k x \sin \alpha)+\sin \left(k \sin \alpha\left(-\frac{x}{2}-y \frac{\sqrt{3}}{2}\right)\right) \\
-\sin \left(k \sin \alpha\left(-\frac{x}{2}+y \frac{\sqrt{3}}{2}\right)\right)
\end{array}\right)^{2}
\end{array}\right] .
$$

Thus, during irradiation of solid surface by several laser beams in accordance with the interference laws a spatially periodic distribution of light intensity $J$ is formed on it. 


\section{The laser-induced grating of the refraction index in semiconductors}

Let us consider a condensed matter (semiconductor), an outer surface of which is irradiated by two coherent light beams (CLBs) with an equal frequency that satisfies the inequality, $\bar{\varepsilon}<<\hbar \omega<<\varepsilon_{g}$ ( $\bar{\varepsilon}$ is the carrier average energy and $\varepsilon_{g}$ is the bandgap of this semiconductor). These CLBs, as known [2-10], form an interference pattern on a condensed matter surface an interference pattern (Fig. 1). Let us look, how the CLBs can influence on the refractive index of semiconductor. As the energy of the CLB quanta is less than the forbidden gap value, $\hbar \omega<\varepsilon_{g}$, then the oscillations of charge carriers (electrons) do not happen, and only their redistribution under the CLBs action is observed. The electrons will move from the illuminated areas to the dark ones, reserving regions of a positive charge. This causes an appearance of electric field between areas of positive and negative charges. Its action gives rise to a strain of a semiconductor crystalline lattice. This strain is the reason for light to move faster through one area and move slowly through another one. It implies periodical changes in the refraction index. Thus, we obtain the spatially-periodic laser-induced structure - the grating of the refractive index of semiconductor. The laser-induced grating of the refractive index obtained in such manner will be shifted in space by a quarter of the period in comparison with an interferential picture created by the CLBs on the semiconductor surface.

Now some analytical calculations confirming occurrence of the laser-induced grating of the refractive index in the semiconductor will be presented. As it is known, all the substances are characterized by the complex refraction index $N$ that determines a decrease of the light velocity inside the substance in comparison with that in empty space

$N=c \sqrt{\varepsilon \mu}$,

where $\varepsilon$ and $\mu$ are dielectric and magnetic permeabilities of semiconductor, respectively. These quantities are related (in linear approximation) to the medium properties by the following relations

$\varepsilon=1+4 \pi \chi_{e}, \mu=1+4 \pi \chi_{\mu}$,

where $\chi_{e}$ and $\chi_{\mu}$ are dielectric and magnetic susceptibilities of semiconductor, respectively. Substituting (12) into (11), the following expression for the complex refractive index is obtained

$N^{2}=c^{2}\left(1+4 \pi \chi_{e}\right)\left(1+4 \pi \chi_{\mu}\right)$.

First, we calculate $\varepsilon$ for a semiconductor in the high frequency field of the CLBs, using the well known Lorentz model. In this model, condensed matter is considered as a set of one-electron atoms. The movement of an electron in each atom is featured in the approach of elastic interaction of the electron with the nucleus. The force of their interaction is considered to be proportional to the electron shift from its equilibrium position in the absence of external field $f=-q r$. Here, $q(q>0)$ is the coupling constant, and the negative sign means that the elastic force arising from the electron shift is directed oppositely to the shift. Instantaneous coordinate of an electron $\vec{r}$ that is under action of the force $f_{E}=-e E$, caused by the CLBs can be determined from the second Newton law

$$
m \frac{d^{2} \vec{r}}{d t^{2}}=f+f_{E},
$$

where $m$ and $e$ are the mass and charge of an electron, respectively. Taking into account in (14) the force, which brakes an electron, $f_{t}=2 \gamma \vec{r} \quad(\gamma-$ some constant factor), the expression (14) yields

$\frac{d^{2} \vec{r}}{d t^{2}}+\left(2 \gamma+\omega_{0}^{2}\right) \vec{r}+\frac{e}{m} \vec{E}=0$,

where $\omega_{0}^{2}=q / m$.

The electric intensity of the light wave $E$ propagating in semiconductor can be found from the Maxwell equations. For the simplicity, we are restricted by the one-dimensional case

$\frac{\partial^{2} E}{\partial t^{2}}=\frac{\varepsilon}{c^{2}} \frac{\partial^{2} E}{\partial t^{2}}$

where the dielectric constant of semiconductor $\varepsilon$ is determined by the dipole moment quantity induced by the field of the CLBs:

$\varepsilon=4 \pi \vec{P} / \vec{E}$.

The dipole moment $\vec{P}$ in the Lorentz model is equal to the product of an electron charge $(-e)$, an electron shift $\vec{r}$, described by the equation (15), and the electron concentration $n$

$\vec{P}=-n e \vec{r}$.

Thus, Eqs. (15) and (16) form the system of the equations for the determination of $\vec{r}(t)$ and $\vec{E}(t)$. The direct substitution yields

$$
\vec{E}=\vec{E}(\omega) e^{i \omega t}+c . c . ; \quad \vec{r}=-\frac{e \vec{E}(\omega)}{m\left(\omega_{0}^{2}+2 i \gamma \omega-\omega^{2}\right)}+c . c .
$$

where c.c. means complex conjugate quantity to the first addend.

Substituting (19) into (18), the expression for the polarization vector $\vec{P}$ can be obtained

$$
\vec{P}=\frac{n e^{2} \vec{E}(\omega) e^{i \omega t}}{m\left(\omega_{0}^{2}+2 i \gamma \omega-\omega^{2}\right)}+c . c .
$$

Substitution of Eq. (20) into Eq. (17) gives the following expression for the dielectric constant of semiconductor at the frequency $\omega$

$\varepsilon(\omega)=1+\frac{n e^{2}}{m}\left(\omega_{0}^{2}+2 i \gamma \omega-\omega^{2}\right)^{-1}$. 
The change of the semiconductor refraction index $\Delta N$ in the CLBs field can be easily obtained from Eq. (14) under the conditions $\omega>>\omega_{0}, \omega>>2 \gamma$,

$\Delta N \approx \frac{n e^{2}}{2 \bar{N} m \omega^{2}}$.

It is known $[13,14]$ that at $\bar{\varepsilon}<<\hbar \omega<<\varepsilon_{g}$ the

CLBs in semiconductors redistribute the bulk charge carriers creating a static laser-induced grating of charge carriers concentration. In other words, in this case, the concentration of charge carriers can be defined as $n=n_{0}\left(1+\xi_{1} \cos 2 k_{1 z} z+\xi_{2} \sin 2 k_{1 z} z\right)$

(where $n_{0}$ is the concentration of electrons in semiconductor in the absence of the CLBs field). The amplitudes $\xi_{1}$ and $\xi_{2}$ depend on both the CLBs electric field amplitude and the semiconductor parameters. The method of their calculation can be found in $[13,14]$.

Using Eqs. (22) and (23), the following expression for the non-linear component of the semiconductor refraction index caused by the redistribution of charge carrier concentration in the field of the CLBs can be obtained

$\Delta N \approx \frac{e^{2} n_{0}\left(1+\xi_{1} \cos 2 k_{1 z} z+\xi_{2} \sin 2 k_{1 z} z\right)}{2 \bar{N} m \omega^{2}}$,

where $\bar{N}$ is the average value of the semiconductor refractive index.

Thus, it follows from (24) that the change of the semiconductor refraction index caused by redistribution of charge carrier concentration in the CLBs field will have spatially periodic modulation, i.e. in the semiconductor in the interference field of the CLBs a laser-induced grating of the refraction index is formed.

Since the absorption index of light in semiconductor $\alpha$ is proportional to the carrier concentration as

$\alpha \approx \frac{2 \pi e^{2} \tau_{0} n_{0}\left(1+\xi_{1} \cos 2 k_{1 z} z+\xi_{2} \sin 2 k_{1 z} z\right)}{c \sqrt{\varepsilon_{0}} m}$

( $\tau_{0}$ is the electron free path time), so it will be also spatially modulated in a periodic manner.

So, the optical parameters of the media $n$ and $\alpha$, on the surface of which the interference pattern is created change according to the interference pattern intensity distribution $J$ that gives rise to the spatially periodic modulation of $n$ and $\alpha$, what is the reason for possible occurrence of diffraction of additional falling light beams [15].

\section{Diffraction on a thin grating of the refraction index in semiconductors}

Let us consider the diffraction of the falling electromagnetic wave on the laser-indused grating of the refraction index of the thickness $z_{0}$ that is much greater than the length of the falling light wave $\lambda$. In this case, the variation of the falling wave amplitude on the coordinate can be assumed smooth, and the second-order derivative of the amplitude with respect to the coordinate along the wave propagation is negligible in comparison with the first-order derivative. Let us assume that a flat electromagnetic wave propagates along the $O Z$ axis

$\vec{E}=\vec{E}_{0} \exp \{i(\omega t-\vec{k} \bar{r})\}+c . c$.

(where c.c. means complex conjugate quantity to the first addend, the constant component of the phase $\varphi_{0}$ is included in the value of $E_{0}$ ), the "lines" of the grating are directed perpendicular to the $O Z$ axis, the polarization vector of the wave is directed along the $O Y$ axis (Fig. 2). When $z_{0}<<L$ ( $L-$ the grating period), in the Maxwell equation

$\nabla \times(\nabla \times \vec{E})=-\frac{1}{c^{2}} \frac{\partial^{2} \vec{D}}{\partial t^{2}}-\frac{4 \pi}{c^{2}} \frac{\partial \vec{j}}{\partial t}$

the derivative with respect to $x$, in comparison with the derivative with respect to $z$, can be neglected. So, in assumption of amplitude smoothness, substitution of Eq. (25) into Eq. (26) gives:

$\frac{\partial E_{0}}{\partial z}=-\frac{i}{2 k}\left(\frac{\omega^{2}}{c^{2}}-\varepsilon-2 i \frac{\omega}{c} \sqrt{\varepsilon \alpha}\right) E_{0}$.

The values of the parameters $\alpha$ and $\varepsilon$ are considered to be the spatially periodic functions of $x$ coordinate that can be written as

$\varepsilon=\varepsilon_{0}+\varepsilon_{1} \cos q x, \quad \alpha=\alpha_{0}+\alpha_{1} \cos q x, \quad q=\frac{2 \pi}{L}$.

We are restricted by the case of small modulation of the $\varepsilon$ and $\alpha$ values, Eq. (27) yields

$\frac{\partial E_{0}}{\partial z}=-\frac{i}{2 k}\left(-\alpha_{0}-\left(i \gamma^{\prime}+\gamma^{\prime \prime}\right) \cos q x\right) E_{0}$,

where we use $\gamma^{\prime}=\frac{\omega \varepsilon_{1}}{2 c \sqrt{\varepsilon_{0}}}, \gamma^{\prime \prime}=\alpha_{1}$.

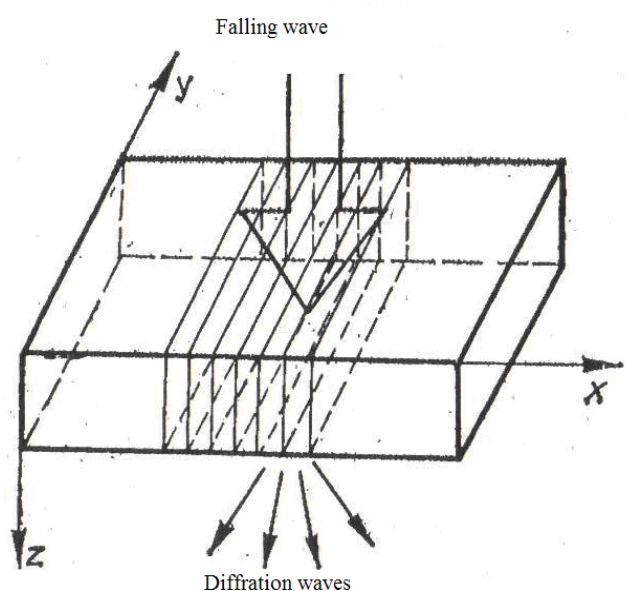

Fig. 2. Scheme of the Rahman-Nat diffraction on a thin grating. 
The solution of Eq. (29) is as follows

$E_{0}(x, z)=T(x, z) E_{0}(0)$,

where $E_{0}(0)$ is the amplitude of a wave falling on the illuminated surface $z=0$ of the grating, and the value of $T(x, z)$ is the function of the transmission that can be written as

$$
T(x, z)=\exp \left\{-\alpha_{0} z\right\} \cdot \exp \left[-\left(\gamma^{\prime \prime}+i \gamma^{\prime}\right) z \cos q x\right] .
$$

As follows from Eq. (31), the amplitude of a beam passing through a thin grating $\left(z_{0}<<L\right)$ is defined as a simple product of the initial amplitude $E_{0}(0)$ and a function of the grating transmission $T$.

The transmission function $T(x, z)$ can be easily presented as the Fourier series, the coefficients of which are obtained from Eq. (31),

$$
\begin{aligned}
& T(x, z)=\sum_{l} T_{l}(z) \exp \{\text { ilq } x\}, \\
& T_{l}(z)=\sum_{m} i^{(l-m)} I_{m}\left(\alpha_{1}, z\right) J_{l-m}\left(\gamma^{\prime} z\right) e^{-\alpha_{0} z}(-1)^{m},
\end{aligned}
$$

where the indexes $l$ and $m$ take the integer values $0, \pm 1, \pm 2, \ldots$ up to infinity; $J_{m}-$ the Bessel function, $I_{m}$ - the Bessel function of the imaginary argument.

In the case of purely phase grating $\alpha_{1}=0$, so in the sum with respect to $m$ in Eq. (32) only the term with $m=0$ is left, and the transmission function will be written as

$$
T_{l}(z)=i^{l} J_{l}\left(\gamma^{\prime} z\right) e^{-\alpha_{0} z}
$$

For a purely amplitude grating, when $\gamma^{\prime}=0$, in the sum with respect to $m$ in Eq. (32) only the term with $m=l$ is left, and the transmission function will be written as

$$
T_{l}(z)=I_{l}\left(\alpha_{1} z\right) e^{-\alpha_{0} z} .
$$

Thus, in accordance with Eqs (30) and (32), a flat electromagnetic wave (25), propagating through the laser-induced diffraction grating of the refraction index is converted into a set of flat waves $e^{i(k z+l q x)}$

(Fig. 2).

In this case, one of the waves with $l=0$ passes through the grating without change (the zero order of diffraction), and the others are the maxima of the higher orders when the intensity decreases with the increase of $|l|$. The diffraction of this type is called the Rahman-Nat diffraction. It is worth to mention that the intensity distribution in the maxima is determined by the grating type (amplitude or phase) and its thickness $z_{0}$. The diffraction pattern is numerically described by the ratio of the intensity of diffraction wave $J_{l}=\left|E_{l}\right|^{2}$ to the intensity of the falling wave $J_{0}=\left|E_{0}\right|^{2}$, which is called by the efficiency of the diffraction grating

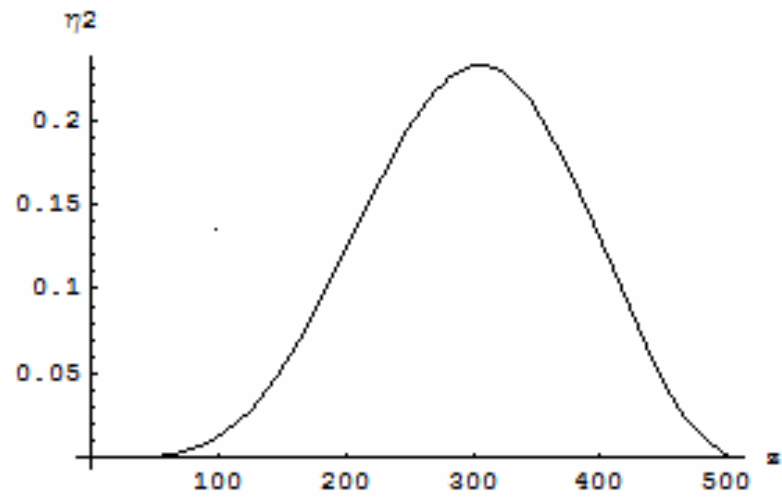

Fig. 3. The dependence of the diffraction efficiency of the phase grating on its thickness value.

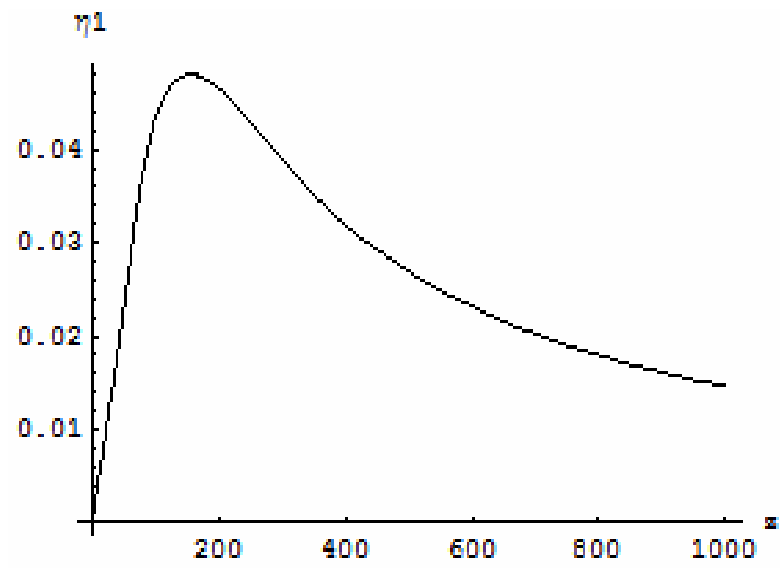

Fig. 4. The dependence of the diffraction efficiency of the amplitude grating on its thickness value.

$\eta_{l}=\frac{\left|E_{l}\right|^{2}}{\left|E_{0}\right|^{2}}=\left|T_{l}\right|^{2}$.

For the phase grating in accordance with Eq. (33), the diffraction efficiency is equal to

$\eta_{l}=J_{l}^{2}\left(\gamma^{\prime} z\right) e^{-2 \alpha_{0} z}$

and for the amplitude grating in accordance with Eq. (34) we have

$\eta_{l}=I_{l}^{2}\left(\alpha_{1} z\right) e^{-2 \alpha_{0} z}$.

In Figs 3 and 4, the dependence produced by numerical calculations for the diffraction efficiency of the phase $\eta_{2}$ and amplitude $\eta_{1}$ gratings on the free carriers that are created by the interference pattern from two coherent laser beams on the thickness of grating $z$ it is shown. As one can see the value of the maximal diffraction efficiency for the Rahman-Nat diffraction of the first order is approximately equal to the $28.2 \%$ for the purely phase grating at the thickness value $z \sim 200$ and to the $4.8 \%$ for the purely amplitude grating at the thickness value $z \sim 150$. In accordance with Eqs (33) and (34), the waves belonging to the nearby diffraction orders differ from each other by $\pi / 2$ in their phase. 


\section{Conclusion}

In this paper, it is shown that on the laser induced grating of the refraction index on the free charge carriers that is created as a result of illumination of semiconductor surface by symmetrically oriented laser beams, the Rahman-Nat diffraction can be observed. Numeral calculations performed by us show that the maximal diffraction efficiency for the Rahman-Nat diffraction of the first order is $28.2 \%$ for a purely phase grating at its thickness $z \sim 200$ and $4.8 \%$ for a purely amplitude grating. It is also worth mentioning that waves belonging to the nearby diffraction orders differ from each other by $\pi / 2$ in their phase.

\section{References}

1. M.J. Birnbaum, Semiconductor surface damage produced by ruby lasers // Appl. Phys. 36 (11), p. 3688-3691 (1965).

2. N.I. Koroteev, I.L. Shumay, Physics of High-Power Laser Radiation. Nauka, Moscow, 1991 (in Russian).

3. S.A. Akhmanov, V.I. Emelianov, N.L. Koroteev and V.N. Seminog, Influence of high-power laser radiation on the surface of semiconductors and metals: non-linear optic effects and non-linear optic diagnostics // Uspekhi fiz. nauk, 147 (4), p. 675-745 (1985) (in Russian).

4. A. Lasgni, C. Holzapfel, and F. Muklich, Periodic pattern formation of intermetallic phases with long range order by laser interference metallurgy // $A d v$. Eng. Matter. 7 (6), p. 487-491 (2005).

5. Y. Lu, J. Barhen, Y. Braiman, and J.X. Zhong, Interference pattern from an array of coherent laser beams // J. Vac. Sci. Technol. B 20(6), p. 2602-2605 (2002).

6. F. Muklich, F. Lasagni, and C. Daniel, Laser interference metallurgy - periodic surface pattering and formation of intermetallics // Intermetalics 13, p. 437-442 (2005).
7. C. Daniel, F. Muklich, and Z. Liu, Periodical micro-nano-structuring of metallic surfaces by interfering laser beams // Appl. Surf. Sci. 208-209, p. 317-321 (2003).

8. F. Yu, P. Li, and F. Muklich, Laser interference lithography as a new and efficient technique for micropatterning of biopolymer surface // Biomaterials, 26, p. 2307-2312 (2005).

9. M. Veith, C. Daniel, and F. Muklich, Periodical micro-structuring of hydride containing metastable aluminum oxide using laser interference metallurgy // Adv. Eng. Mat. 7 (1-2) p. 27-29 (2005).

10. K.W. Liu, C. Gachot, and F. Muklich, Combinatorial experiment in Ni-Ti thin films by laser interference structuring // Appl. Surf. Sci. 247, p. 550-555 (2005).

11. O.Yu. Semchuk, V.N. Semioshko, R.V. Bila, M. Willander and M. Karlsteen, Refractive index of ferromagnetic semiconductors in a field of coherent light beams // Functional Materials 11 (1), p. 159163 (2004).

12. O.Yu. Semchuk, V.N. Semioshko, L.G. Grechko, M. Willander and M. Karlsteen, Laser ablation lithography on thermoelectric semiconductor // Appl. Surf. Sci. 252, p. 4759-4762 (2006).

13. A.E. Levshin, O.Yu. Semchuk, and P.M. Tomchuk, Superlattices formed in ferromagnetic semiconductors by coherent light beams // Sov. Phys.Solid State 28 (2) 229-232 (1986).

14. I.M. Dykman, P.M. Tomchuk, Influence of the coherent light beams on the free carrier in semiconductors // Fizika tverd. tela 26 (9), p. 27292733 (1984) (in Russian).

15. O.Yu. Semchuk, L.G. Grechko, Light diffraction in a ferromagnetic semiconductor with a superlattice on nonequilibrium electrons and magnons // Ukrainsky fizychny zhurnal 38 (8), p. 1157-1163 (1993) (in Ukrainian). 